\title{
钛酸酯偶联剂对包硅铝钛白粉表面的有机改性 *
}

\author{
林玉兰王亭杰覃操金涌 \\ (清华大学化学工程系, 北京 100084)
}

关键词：钛白粉，钛酸酯，表面改性，疏水

由于钛白粉具有独特的物理化学性质和优良的 颜料性能, 被广泛地应用在涂料、塑料、合成纤维、橡 胶和油墨等行业. 但未经表面改性的钛白粉颗粒, 在涂料介质中的分散性较差, 加之其较强的光化学 活性, 使得其颜料应用性能较差。因此, 必须对其进 行改性。其表面改性可分为无机改性和有机改性 . 无机改性可提高耐候性, 常用的改性剂为硅、铝氧化 物 ${ }^{[1,2]}$. 有机改性可有效的改进钛白粉在介质中的 润湿性和分散性, 常用的改性剂为有机胺及其盐 ${ }^{[3]}$ 、

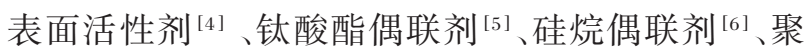
合物 ${ }^{[7]}$ 及 $\mathrm{DWA}^{[8]}$ 等。

金红石型钛白粉表面经过硅铝 $\left(\mathrm{SiO}_{2} 、 \mathrm{Al}_{2} \mathrm{O}_{3}\right)$ 双 层包覆后, 具有较好的耐侯性. 但由于其表面亲水 特性, 在非水体系中的分散性较差, 从而影响了它在 高档油漆、填料等行业中的应用. 因此, 高档钛白粉 必须进行有机改性. 本文针对平均粒径为 $0.3 \mu \mathrm{m}$ 的金红石型钛白粉颗粒, 在硅铝双层包覆的基础 上 ${ }^{[9]}$, 用钛酸酯偶联剂对其表面进行有机改性.

\section{1 实 验}

\section{1 实验试剂}

钛酸酯偶联剂 CA6[NDZ101, $\left(\mathrm{CH}_{3}\right)_{2} \mathrm{CHOTi}-$ $\left.(\mathrm{OCOOR})_{2} \mathrm{OP}(\mathrm{O})\left(\mathrm{OR}^{\prime}\right)_{2}\right] 、 \mathrm{CA} 7 \mathrm{NDZ} 201,\left(\mathrm{CH}_{3}\right)_{2} \mathrm{CHOTi}-$ $\left(\mathrm{OP}(\mathrm{O})(\mathrm{OH}) \mathrm{OP}(\mathrm{O})\left(\mathrm{OC}_{8} \mathrm{H}_{17}\right)_{2}\right)_{3}$ ] (南京曙光化工一 厂) 为工业级产品; 异丙醇 (北京益利精细化学品有 限公司) 为分析纯试剂; 未包覆改性的金红石型 $\mathrm{TiO}_{2}$ (平均粒径 $0.3 \mu \mathrm{m}$, 镇江钛白粉厂) 为工业级 产品.

\section{2 样品制备}

自制 $\mathrm{SiO}_{2} 、 \mathrm{Al}_{2} \mathrm{O}_{3}$ 双层包覆 $\mathrm{TiO}_{2}{ }^{[9]}$, 包覆层厚度 各为 $5 \sim 10 \mathrm{~nm}$. 将占颗粒重量 $2 \%$ 的钛酸酯偶联剂 溶解在异丙醇中, 加人无机包覆后的 $\mathrm{TiO}_{2}$ 颗粒, 其 浓度为 $20 \%$ (质量分数). 搅拌 $1 \mathrm{~h}$, 过滤干燥, 用异 丙醇溶剂进行 $8 \sim 9 \mathrm{~h}$ 索式抽提. 干燥后, 制成样品. 通过抽提脱附颗粒表面物理吸附的包覆剂, 以便确 定包覆剂与颗粒表面的作用方式. 在无钛酸酯存在 的条件下, 可制备抽提的 $\mathrm{TiO}_{2}$ 颗粒样品, 用于对比 分析. 针对 CA6、CA7 改性剂, 样品分别记作 $\mathrm{TiO}_{2}-\mathrm{CA} 6 、 \mathrm{TiO}_{2}-\mathrm{CA}$, 用于测试分析.

\section{3 测试分析}

将有机改性前后的 $\mathrm{TiO}_{2}$ 粉末经 200 目篮分后 分散在水中, 观察和评价其疏水特性; 采用 NICOLET-5DX 型傅立叶变换红外光谱仪分析样品表面 的官能团; 采用 PYR-2A 型气相色谱用热分解器及 GC-14B 气相色谱仪进行样品的高温裂解色谱分析 (GDX-502 柱), 检测样品中的微量有机物, 裂解温 度 $600{ }^{\circ} \mathrm{C}, \mathrm{N}_{2}$ 载气 (流量 $35 \mathrm{~mL} \cdot \mathrm{min}^{-1}$ ), FID 检测 器; 以 TGA2050 型热重分析仪检测颗粒表面有机 物的包覆量; PHI-5300/ESCA 型 XPS 谱仪分析改 性剂与颗粒表面的化学键合结构, $\mathrm{Al}$ 阳极靶, 分析 室真空度 $2.9 \times 10^{-7} \mathrm{~Pa}$.

\section{2 结果与讨论}

\section{1 改性效果}

将钛酸酯有机改性前后的 $\mathrm{TiO}_{2}$ 粉末样品分散 到水中, 根据颗粒在水中的沉浮现象, 定性表征颗粒 的疏水特性. 实验中观察到, 未经有机改性的 $\mathrm{TiO}_{2}$ 粉末呈现出显著的亲水性; 而经钛酸酯改性后的 
表 1 有机改性前后样品的裂解色谱分析 $\left(600{ }^{\circ} \mathrm{C}\right)$

Table 1 Sample analysis by pyrolytic gas chromatography $\left(600{ }^{\circ} \mathrm{C}\right)$

\begin{tabular}{cccccccccc}
\hline & $\mathrm{CH}_{4}$ & $\mathrm{C}_{2} \mathrm{H}_{4}$ & $\mathrm{C}_{2} \mathrm{H}_{6}$ & $\mathrm{C}_{3} \mathrm{H}_{6}$ & $\mathrm{CH}_{3} \mathrm{OH}$ & $\mathrm{CH}_{3} \mathrm{CHO}$ & $\Sigma, \%$ & $\Sigma \mathrm{C}, \%$ \\
\hline $\mathrm{TiO}_{2}$ & 0.0000 & 0.0000 & 0.0000 & 0.0000 & 0.0000 & 0.0000 & 0.0000 & 0.0000 \\
Extracted $\mathrm{TiO}_{2}$ & 0.0016 & 0.0013 & 0.0017 & 0.0072 & 0.0118 & 0.0115 & 0.0351 & 0.0205 \\
$\mathrm{TiO}_{2}-\mathrm{CA} 7$ & 0.0030 & 0.0132 & 0.0019 & 0.0324 & 0.0154 & 0.0154 & 0.0812 & 0.0569 \\
\hline
\end{tabular}

express concentration as mass percent

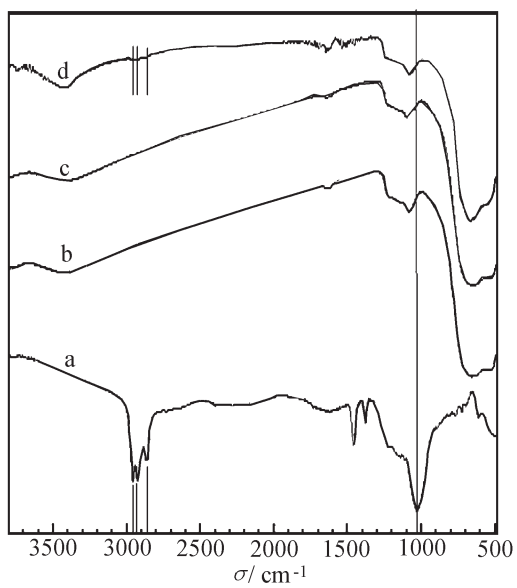

图 $1 \mathrm{TiO}_{2}$ 及 $\mathrm{TiO}_{2}-\mathrm{CA} 7$ 的红外吸收光谱

Fig. 1 The IR absorption spectra of samples

a: CA7, b: $\mathrm{TiO}_{2}$, c: Extracted $\mathrm{TiO}_{2}$, d: $\mathrm{TiO}_{2}-\mathrm{CA} 7$

$\mathrm{TiO}_{2}$ 粉末悬浮在水面上, 不沉降, 充分摚拌后仍然 悬浮于水面, 具有较强的疏水性。后一现象表明 $\mathrm{TiO}_{2}$ 颗粒表面已被钛酸酯分子比较牢固包裹着. 由于样品是经过长时间抽提的, 其表面偶联剂包覆 层仍不被溶剂所萃取, 说明钛酸酯偶联剂与 $\mathrm{TiO}_{2}$ 的 表面包覆层可能发生了化学键合作用。

\section{2 红外光谱分析}

将 CA7、未经有机改性的 $\mathrm{TiO}_{2}$ 抽提前后及有机 改性并抽提的 $\mathrm{TiO}_{2}-\mathrm{CA} 7$ 样品进行红外光谱分析, 结果见图 1. 由图可见, $\mathrm{TiO}_{2}-\mathrm{CA} 7$ 样品在 2956 、 $2927 、 2860 \mathrm{~cm}^{-1}$ 处出现 $\mathrm{CH}_{3} 、 \mathrm{CH}_{2}$ 基团的特征吸收 峰(这是 $\mathrm{CA} 7$ 中的 $\mathrm{CH}_{3} 、 \mathrm{CH}_{2}$ 基团的吸收峰); 而未 经有机改性 $\mathrm{TiO}_{2}$ 样品抽提前后的谱图相同, 不存在 二基团的特征吸收峰。说明在抽提过程中, 异丙醇 在颗粒表面的化学吸附很少或未与颗粒表面反应, 有机改性后的颗粒表面存在 CA7. CA7 的特征吸收 峰为 $1032 \mathrm{~cm}^{-1}, \mathrm{TiO}_{2}$ 在 $1086 \mathrm{~cm}^{-1}$ 处出现吸收峰, 抽提后的 $\mathrm{TiO}_{2}-\mathrm{CA} 7$ 在 $1084 \mathrm{~cm}^{-1}$ 处出现吸收峰, 吸 收峰偏移了 $2 \mathrm{~cm}^{-1}$. 限于红外光谱的分辨率, 虽不 足以说明 $\mathrm{CA} 7$ 与颗粒发生键合, 但由于 $\mathrm{CH}_{3} 、 \mathrm{CH}_{2}$ 吸收峰的存在, 说明 CA7 与颗粒之间可能发生了键
合。偶联剂中其他有机官能团吸收峰由于偶联剂用 量 $(2 \%)$ 太少而未能表现出来. 抽提后样品的 $\mathrm{CH}_{3}$ 、 $\mathrm{CH}_{2}$ 基团吸收峰稍为减弱, 这是因为样品经抽提后, 没有发生键合、只发生吸附的那部分偶联剂 CA7 被 萃取. 因此, 可以认为 $\mathrm{CA} 7$ 与 $\mathrm{TiO}_{2}$ 表面包覆层之 间可能存在化学键合作用。

\section{3 高温裂解色谱分析}

通过高温气相裂解色谱可以检测样品中的微量 有机物. 表 1 是在 $600{ }^{\circ} \mathrm{C}$ 下通过气相裂解色谱对有 机改性前后的样品 $\mathrm{TiO}_{2}$ 及 $\mathrm{TiO}_{2}-\mathrm{CA} 7$ 的分析结果. 由表可见, 有机改性前的 $\mathrm{TiO}_{2}$ 在此温度下基本不发 生裂解, 而在同样条件下 $\mathrm{TiO}_{2}-\mathrm{CA} 7$ 发生明显的裂 解. 为检验异丙醇在长时间抽提过程中, 是否与颗 粒表面的羟基发生反应, 对有机改性前 $\mathrm{TiO}_{2}$ 在相同 条件下经过异丙醇抽提后的样品, 进行裂解色谱分 析, 发现有明显的裂解产物, 其成分与含量见表 1 . 表 1 表明在抽提过程中异丙醇与表面羟基发生了反 应. 尽管如此, 表 1 中仍显示出 CA7 改性后的裂解 产物显著高于无钛酸酯存在条件下抽提的 $\mathrm{TiO}_{2}$, 因 此也说明改性剂 $\mathrm{CA} 7$ 与 $\mathrm{TiO}_{2}$ 颗粒表面的结合比较 牢固, 并有一定的包覆量 .

\section{$2.4 \mathrm{X}$ 光电子能谱分析}

采用 XPS 谱仪对有机改性前后样品 $\mathrm{TiO}_{2}$ 及 $\mathrm{TiO}_{2}-\mathrm{CA} 7$ 进行表面成分分析, 发现改性后并经长时 间抽提的颗粒表面存在元素磷 (改性剂中含有磷), 证实改性颗粒表面存在钛酸酯偶联剂。

表 2 列出有机改性前后 $\mathrm{TiO}_{2}$ 的 XPS 图谱峰位. 从表 2 可以看出, 未经有机改性的样品中的 $\mathrm{Al} 2 p$ 轨道的电子结合能为 $74.25 \mathrm{eV}$, 有机改性后样品中

\section{表 2 有机改性前后 $\mathrm{TiO}_{2}$ 的 XPS 图谱峰位}

Table 2 The electronic binding energy of the elements on the surface of $\mathrm{TiO}_{2}$ particles from XPS

\begin{tabular}{ccccc}
\hline Samples & Ti $2 p$ & P $2 p$ & Si $2 p$ & Al $2 p$ \\
\hline Pre-modification (eV) & 458.45 & - & 103.05 & 74.25 \\
Post-modification (eV) & 458.40 & 133.65 & 103.00 & 74.00 \\
\hline
\end{tabular}




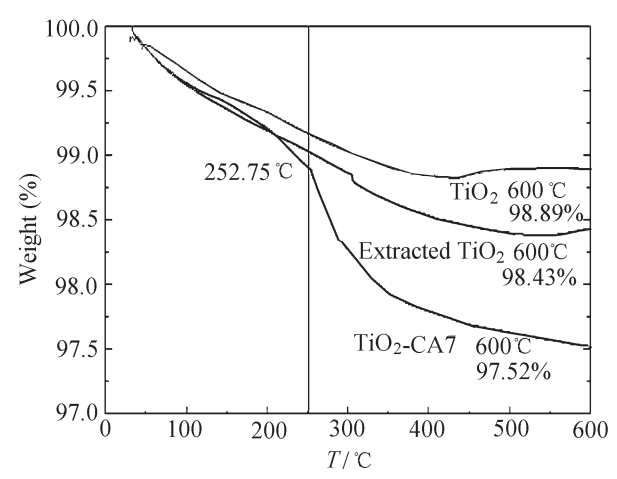

图 2 未改性 $\mathrm{TiO}_{2}$ 及 $\mathrm{TiO}_{2}-\mathrm{CA} 7$ 的 TGA 谱图

Fig. 2 The thermogravimetric curve of $\mathrm{TiO}_{2}$ and $\mathrm{TiO}_{2}-\mathrm{CA} 7$

的 $\mathrm{Al} 2 p$ 轨道的电子结合能为 $74.00 \mathrm{eV}$, 改性后 $\mathrm{Al}$ 的电子结合能比改性前的减少 $0.25 \mathrm{eV}$. 可能是 CA7 中的 $\mathrm{Ti}$ 通过化学键结合于 $\mathrm{TiO}_{2}$ 表面的 $\mathrm{SiO}_{2}$ 、 $\mathrm{Al}_{2} \mathrm{O}_{3}$ 包覆层, 形成了 $\mathrm{Al}-\mathrm{O}-\mathrm{Ti}$ 键. $\mathrm{Al}$ 的电负性 比 $\mathrm{Ti}$ 的电负性略大, 故 $\mathrm{Al}$ 周围的电子密度增大, 屏 蔽效应增大, 导致 $\mathrm{Al}$ 的电子结合能有所减小.

\section{5 热重分析}

在较高温度下, 钛酸酯偶联剂会发生氧化或热 裂解反应. 如果 $\mathrm{TiO}_{2}$ 样品表面存在偶联剂, 在热重 分析中, 其重量会随温度升高而减少. 将 $\mathrm{TiO}_{2}$ 粉末 用过量的偶联剂 $\mathrm{CA} 7(20 \%)$ 于 $25{ }^{\circ} \mathrm{C}$ 对 $\mathrm{TiO}_{2}$ 粉末进 行改性 $1 \mathrm{~h}$, 然后用异丙醇索式抽提 $8 \sim 9 \mathrm{~h}$, 经烘干 得到 $\mathrm{TiO}_{2}-\mathrm{CA} 7$ 样品, 与未经有机改性的 $\mathrm{TiO}_{2}$ 样品 一起进行 TGA 分析, 结果如图 2. 由图 2 可见, 未经 有机改性的 $\mathrm{TiO}_{2}$ 样品重量也随温度升高而减少, 在 $363{ }^{\circ} \mathrm{C}$ 时, 重量减少 $1.13 \%$, 在 $363{ }^{\circ} \mathrm{C}$ 以后重量基本 不变, 这是 $\mathrm{TiO}_{2}$ 颗粒表面吸附水和包覆层中水合氧 化硅和水合氧化铝中所含水分的损失所造成的. 由 于在异丙醇抽提过程中, 异丙醇与颗粒表面发生反 应, 故抽提 $\mathrm{TiO}_{2}$ 样品随温度升高也有显著失重. 异 丙醇包覆量约为 $0.46 \%$. $\mathrm{TiO}_{2}-\mathrm{CA} 7$ 的重量随温度 升高而减少, 在 $252{ }^{\circ} \mathrm{C}$ 以前, 其失重曲线与 $\mathrm{TiO}_{2}$ 样 品基本一样, 在 $252{ }^{\circ} \mathrm{C}$ 以后重量快速减少, $600{ }^{\circ} \mathrm{C}$ 时 失重已达 $2.48 \%$. $\mathrm{TiO}_{2}$-CA7 在 $252{ }^{\circ} \mathrm{C}$ 以后重量的 减少, 一部分是由于水分的损失造成的, 另一部分主 要是 $\mathrm{TiO}_{2}$ 颗粒表面的 $\mathrm{CA} 7$ 和异丙醇氧化分解造成 的. 对比 $\mathrm{TiO}_{2}$ 、抽提 $\mathrm{TiO}_{2} 、 \mathrm{TiO}_{2}-\mathrm{CA} 7$ 的 TGA 谱图, 可以确定在该条件下 $\mathrm{CA} 7$ 与 $\mathrm{TiO}_{2}$ 表面牢固包覆的 最大量约为 $0.91 \%$.

\section{6 作用机理分析}

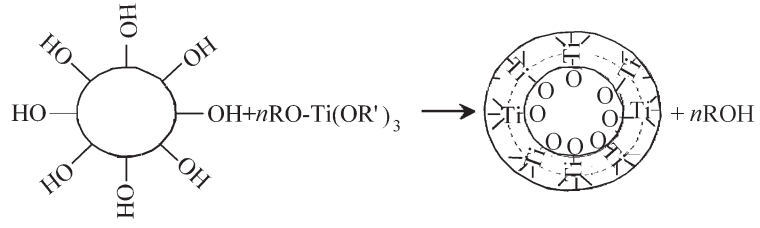

图 3 钛酸酯偶联剂在二氧化钛颗粒表面的作用机理

Fig. 3 The modification mechanism of titanate coupling reagent on the surface of titanium dioxide particles

钛酸酯偶联剂的通式可表示为 $(\mathrm{RO})_{M}-\mathrm{Ti}-(\mathrm{OX}-$ $\left.\mathrm{R}^{\prime}-\mathrm{Y}\right)_{N}$, 其中 $1 \leqslant M \leqslant 4, M+N \leqslant 6 ; \mathrm{R}$ 为短碳链 烷烃基; $R^{\prime}$ 为长碳链烷烃基; $X$ 为 $C 、 N 、 P 、 S$ 等元素; $\mathrm{Y}$ 为羟基、氨基、环氧基等基团。根据偶联剂分子结 构及与颗粒表面的作用机理, 钛酸酯偶联剂可分为 四种基本类型: 单烷氧基型、单烷氧基焦磷酸酯基 型、螯合型、配位体型。CA7 为单烷氧基焦磷酸酯型 钛酸酯偶联剂, 它与颗粒表面的反应可表示为 ${ }^{[10]}$

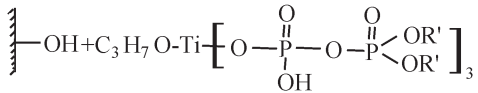

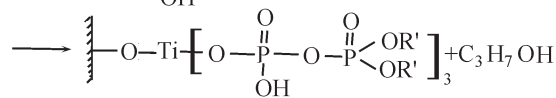

对于微细颗粒, 文献 ${ }^{[11]}$ 提出钛酸酯偶联剂作用的单 分子层模型, 认为典型的钛酸酯偶联剂含有一个异 丙氧基和三个较长的有机长链, 异丙氧基可与颗粒 表面的羟基 (源于颗粒表面的结合水、结晶水、化学 吸附水和物理吸附水) 反应, 形成化学键, 放出异丙 醇, 从而在颗粒表面覆盖了一层单分子膜, 使颗粒表 面特性发生根本性改变。该模型假设：

1. 微细颗粒是一个直径为 $D$ 的球, 颗粒表面 差基分布均匀, 反应能力各向同性;

2. 钛酸酯偶联剂分子是一个以钛离子为中心, 直径为 $d$ 的小圆球体 (不包含三个有机长链), 均匀 地覆盖于微细颗粒表面;

3. 忽略覆盖于颗粒表面的钛酸酯偶联剂分子 之间的作用力和有机长链的位阻效应

基于上述假设, 钛酸酯偶联剂分子在二氧化钛 颗粒表面的作用机理可表示如图 3. 通过在 $\mathrm{TiO}_{2}$ 颗 粒表面形成新的 $\mathrm{Ti}-\mathrm{O}$ 键, 把钛酸酯偶联剂分子与 $\mathrm{TiO}_{2}$ 颗粒结合成一体, 并呈单分子层的分布

由此,可以计算偶联剂的包覆量 ${ }^{[11]}$ :

$$
\frac{W_{0}}{W_{\mathrm{t}}}=\frac{6 \times 10^{-6} \times M}{\rho N_{\mathrm{A}} D d^{2}} \times 100 \%
$$


式中 $W_{0}$ 为偶联剂包覆量; $W_{\mathrm{t}}$ 为颗粒重量 $; N_{\mathrm{A}}$ 为阿 佛加德罗常数 $\left(\mathrm{mol}^{-1}\right) ; \rho$ 为改性颗粒的密度 $(\mathrm{kg} \cdot$ $\left.\mathrm{m}^{-3}\right) ; M$ 为偶联剂的摩尔质量 $\left(\mathrm{g} \cdot \mathrm{mol}^{-1}\right) ; D$ 为改 性颗粒的平均粒径 $(\mathrm{mm}), d$ 为偶联剂分子的计算直 径 $(\mathrm{mm})$, 对于钛酸酯偶联剂, 在数值上等于 $\mathrm{Ti}-\mathrm{O}$ 键长的两倍, 即 $4.16 \times 10^{-6} \mathrm{~mm}$.

由式 (2) 可见, 随着颗粒粒径的减小, 偶联剂的 计算包覆量快速增加. 对于平均粒径为 $300 \mathrm{~nm}$ 二 氧化钛颗粒, 偶联剂的包覆量可达 $10 \%$. 而从 TGA 实验结果分析得到的偶联剂包覆量仅约为 $0.91 \%$, 与用单分子层模型的计算值相差较大. 主要原因是 单分子层模型忽略了包覆在颗粒表面的钛酸酯偶联 剂分子之间的作用力和有机长链的位阻效应, 假设 颗粒表面均布差基并均匀键合钛酸酯偶联剂分子。 而对于亚微米颗粒, 其颗粒表面已不再是均布一层 偶联剂分子, 由于颗粒细小, 覆盖于颗粒表面的偶联 剂分子之间的作用力和有机长链的位阻效应已不可 忽略, 尤其是对于超微细颗粒而言, 需要通过引人位 阻因子来修正由于分子间作用力或长链位阻效应所 造成的误差. 此外, 部分差基与溶剂异丙醇反应, 也 使得实际包覆量降低. 因此, 对于亚微米二氧化钛 改性剂的实际包覆量, 仍需要通过实验来确定.

\section{3 结 论}

用钛酸酯偶联剂对硅铝双层包覆后的 $\mathrm{TiO}_{2}$ 进
行有机改性, 能够使 $\mathrm{TiO}_{2}$ 颗粒由亲水性变为疏水 性, 钛酸酯 $\mathrm{CA} 7$ 与 $\mathrm{TiO}_{2}$ 颗粒表面包覆层形成牢固 的化学键合, $\mathrm{CA} 7$ 在 $\mathrm{TiO}_{2}$ 颗粒表面的最大包覆量约 为 $0.91 \%$.

\section{References}

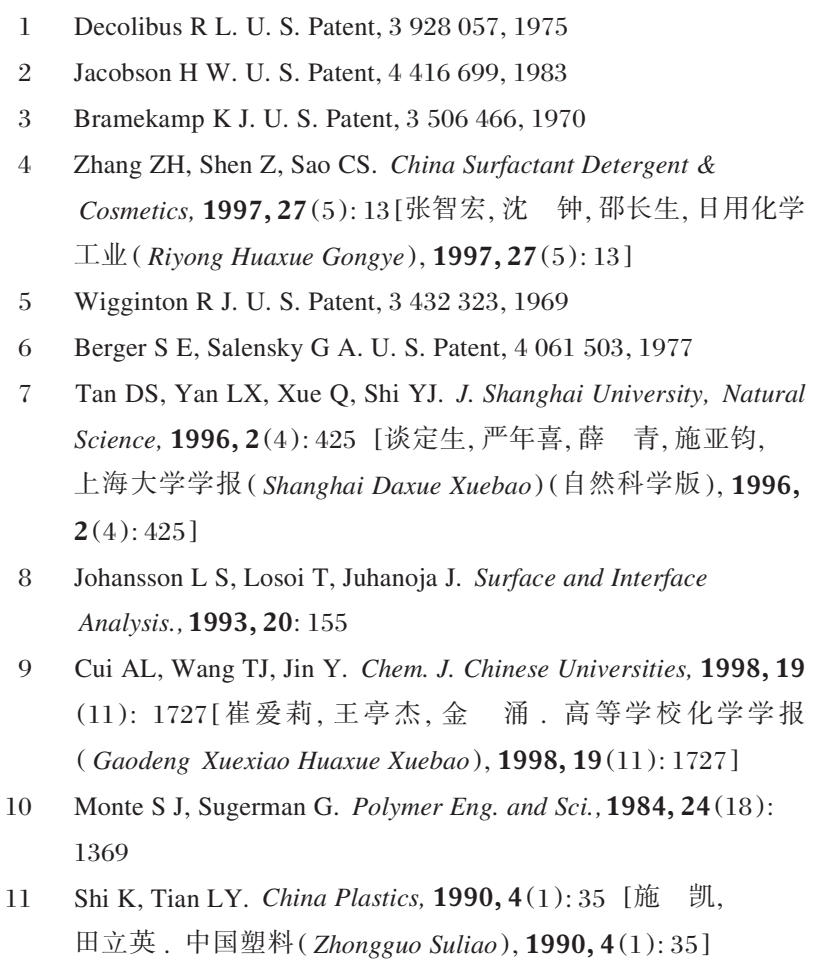

\title{
Surface Organic Modification of $\mathrm{SiO}_{2} \& \mathrm{Al}_{2} \mathrm{O}_{3}$ Coated $\mathrm{TiO}_{2}$ Particles with
} Titanate Coupling Reagent*

Lin Yu-Lan Wang Ting-Jie Q Win Yong

(Department of Chemical Engineering, Tsinghua University, Beijing 100084)

\begin{abstract}
The surface organic modification of $\mathrm{TiO}_{2}$ particles with titanate coupling reagent, which was pre-coated with double layers of $\mathrm{SiO}_{2}$ and $\mathrm{Al}_{2} \mathrm{O}_{3}$, was studied. Experiments showed that the modified particles exhibited hydrophobic characteristics. The modification state of the particle surface was characterized by IR spectroscopic measurement, pyrolytic gas chromatography, thermogravimetric analysis and X-ray photoelectron spectra. The titanate coupling reagent binding with the hydroxyl on the particle surface was analyzed. The surface characteristics of pre-modification and post-modification particles were compared.
\end{abstract}

Keywords: Titanium dioxide, Titanate coupling reagent, Surface modification, Hydrophobic 